\title{
Traceable electric and magnetic field calibrations of radiation monitors for amateur radio inside a parallel plate transmission line up to $30 \mathrm{MHz}$
}

\author{
T. Kootz ${ }^{1}$, T. Schrader ${ }^{2}$, and K. Münter ${ }^{*}$ \\ ${ }^{1}$ DARC - Deutscher Amateur Radio Club, Baunatal, Germany \\ ${ }^{2}$ Physikalisch-Technische Bundesanstalt (PTB), Bundesallee 100, 38116 Braunschweig, Germany \\ * retired, formerly at: DARC and PTB, Germany \\ Correspondence to: T. Kootz (t.kootz@darc.de)
}

\begin{abstract}
In many countries the electromagnetic field strength in the proximity of licensed amateur radio stations is limited to ensure public safety. If the station antenna(s) and/or the environmental situation are complex, only measurement results are acceptable as proof, and proper calibration of the field strength meters ("radiation monitors") is mandatory. A system and a procedure are described here, enabling calibration of such "radiation monitors" at a high quality level and traceable to the SI units, but with low-budget amateur equipment.
\end{abstract}

\section{Introduction}

In many countries the regulations for licensed amateur radio stations require that their emissions are kept below certain public safety limits for the electric $(E)$ and magnetic $(H)$ field strength as well as for the power flux density $(S)$. In Germany the legal situation permits amateur radio operators to file assessments for their stations by themselves. The procedure and requirements are described in a by-law called BEMFV (BNetzA). Further details and how-to informations about station assessment are given in a paper published by the BNetzA, the licensing and supervising authority. Following that paper, for the intended transmitter power and for each antenna system the "safety boundary" must be calculated (a closed surface in 3 dimensions around the antenna), ensuring that outside this boundary no field strength limit for $E, H$ or $S$ will be exceeded. To find this "safety boundary" far-field calculations may be used where appropriate. However, according to BNetzA an alternative approach must be used, if far-field conditions are not applicable. Many amateur radio stations are located in densely populated residential areas, and for short wave frequencies and antennas the far-field conditions can only be expected tens of meters away from the antenna system. But often the neighbour's house is only a few meters away, and in such cases more sophisticated nearfield numerical calculations and precise knowledge of the environment parameters (geometry of objects, their conductivity and susceptibility) would be required, exceeding the capabilities of most radio amateurs. In such a complex situation the field strength measurement with a calibrated instrument is the direct way to a responsible assessment, avoiding any theoretical calculations. But unfortunately a radiation monitor is an expensive instrument for the single radio amateur, required perhaps only once in a few years, and a valid calibration certificate adds even more costs. And not to forget: it takes some practical experience to properly handle a radiation monitor and to obtain reliable results.

Many of the 70000 licensed amateur radio operators in Germany are members of the DARC e.V. (Deutscher Amateur-Radio-Club). This club offers support for their station assessment, especially with software, interpretation sheets, help files, lists of data for all commonly used antenna types, and personal help where required. Over Germany the DARC has 24 districts, subdivided further into about 1000 local groups. All districts and many of those local groups have an appointed EMC expert, who can help members through the station assessment procedure and solve problems as they arise. The 24 EMC experts from the districts meet annually for one weekend to exchange latest know-how and experiences for distribution among their local groups. This 
nationwide multiplier system based on volunteer EMC experts is available to any member anywhere and at minimal cost, but without compromising the quality level, because many of these experts are retired professionals, who have worked in the field of EMC or other electrical sciences.

For this infrastructure the DARC headquarter maintains a few calibrated radiation monitors. Additionally most districts, some local groups and even a few private members have their own instruments, also requiring calibrations in regular intervals. These instruments (approx. 40 devices) are available free of charge for all club members, therefore the cost for annual calibrations in a commercial laboratory is prohibitive. As an alternative, over the recent years the DARC has established and used its own calibration system and procedure with an open parallel-plate transmission line for the shortwave amateur frequency bands below $30 \mathrm{MHz}$.

Although based on proven technology and giving good results, the calibration system was found to be unsatisfactory. The equipment was located and stored at a private site, so the system had to be rebuilt and adjusted for each annual calibration session. A group of amateurs operated it manually, requiring even more time for processing the results and issuing the calibration certificates. During a major revision the equipment was modernized, PC-controlled, finally relocated and installed as a permanent setup at the DARC headquarter. Although no official accreditation as a calibration lab was intended, the quality of installation and procedures should come close to the level required for that.

Continuing public concern about possible adverse health effects of radiofrequency field exposure, the large number of amateur radio installations, and the responsibility of every radio amateur for his activities were the reasons for contacting the PTB (Physikalisch-Technische Bundesanstalt) for support to reach the desired calibration quality level. A cooperation contract defined the framework for the optimization of the DARC calibrations.

\section{Calibration and traceability}

During an usual measurement a physical quantity (e.g. force, temperature, electromagnetic field etc.) of unknown value interacts with a measuring instrument. The exact value of the quantity remains unknown, because even the best instrument has a small, but unavoidable measurement uncertainty. A calibration is also a measurement, but in this case the physical quantity is represented by a suitable "standard" (etalon), so its value is known in advance with only the uncertainty of the standard. The instrument readout is compared with the predefined standard value, and the complete result of the calibration (predefined value with its uncertainty, readout, deviation, uncertainty of the calibrated instrument) are reported in a calibration certificate.

For traceability each calibration laboratory has to maintain "working standards" to provide the physical quantities with predefined value and known uncertainty. The term "traceability" means that these physical quantities are derived from the basic SI physical units by an unbroken chain of calibrations. Under these conditions the calibration result of the laboratory is also "traceable", because there is proof that the instrument under test was compared with a "working standard" linked to the SI units.

Each measured readout value of the instrument must be corrected with the deviation data from the calibration certificate to obtain the "best estimate" for the physical quantity. Its exact value is still unknown, but that "best estimate" is the traceable (!) value coming nearest to it, not the direct readout. Of course, this concept is based on the assumption that deviation and uncertainty of the instrument remain constant over time. Experience has shown that for the DARC radiation monitors a recalibration interval of one year is appropriate.

Because of the immaterial nature of the electromagnetic field quantities there is no standard representing them directly. Instead a system is required here that provides a travelling wave with known electric and/or magnetic field strength and well defined (linear) polarization inside a volume sufficiently large for the radiation monitors to be calibrated. Additionally, the far-field conditions should be valid, i.e. the electric $(E)$ and magnetic $(H)$ field vectors are perpendicular, oscillate in phase, and $E / H=120 \pi \Omega$.

In the radio frequency (rf) range (here between $1.8 \mathrm{MHz}$ and $30 \mathrm{MHz}$ ) resonant radiating antennas are much too large for use inside a room. Instead, TEM-mode transmission lines with suitable geometry and resistive termination are usually preferred as "calculable standards" to produce calibration (far-)fields. e.g. the well-known coaxial transmission cell ("Crawford TEM cell", Crawford, 1974) generates an internal field directly calculable from rf power and cell geometry, but with limited space inside. To be traceable, regular (traceable) recalibration of the cell geometry, the rf power meter, attenuators etc. is required. This direct method generates traceable fields with lowest uncertainty, but is quite expensive and used mainly by national metrology institutes. The PTB maintains such a system (Glimm et al., 1997) for calibration of special "transfer field strength meters" (Münter et al., 1997) with small sensors that can be used elsewhere to reproduce a traceable $E$-field at any external calibration laboratory with moderately increased uncertainty. After the required field strength has been adjusted with the traceable transfer instrument, it is replaced with the radiation monitor to be calibrated. The field generator used for that "substitution method" is quite arbitrary (antennas, transmission lines etc.), it can therefore be very large, and traceability only requires calibration of the transfer field strength meter.

For amateur radio calibration requirements this substitution method seemed appropriate and cost-effective. During the cooperation the PTB provided the calibrated transfer instrument, and the DARC calibration procedure was reviewed under traceability aspects, including the definition of 


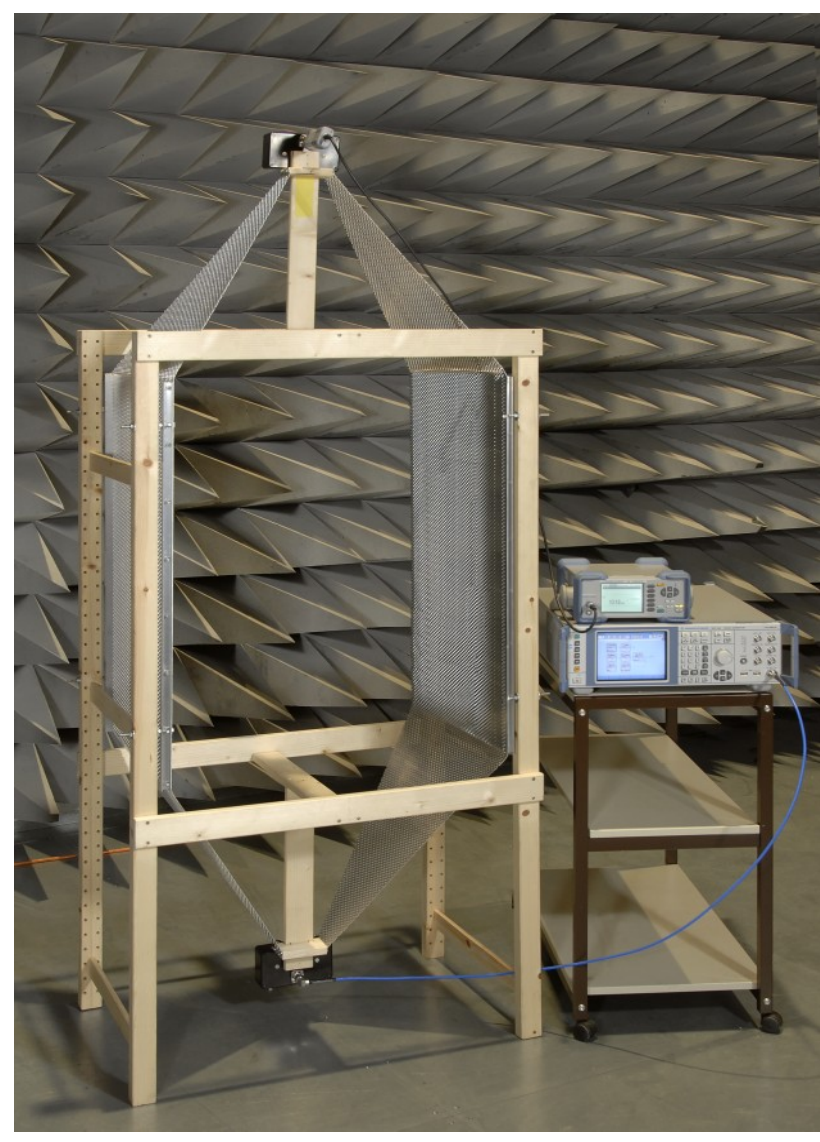

Fig. 1. Balanced Parallel-Plate Line (PTB version).

a convenient method to generate a traceable magnetic $(H$ )field, a topic to be discussed later. The DARC built a second parallel plate line similar to its own device for the PTB.

\section{Parallel-plate transmission line}

For the previous calibrations the DARC made an open, symmetrical parallel plate line with $0.6 \mathrm{~m}$ plate distance and $200 \Omega$ characteristic impedance (see Fig. 1, Parallel-plate line inside the PTB shielded anechoic room). This line offers easy access, sufficient volume, and after successful line impedance optimization it was decided to keep it. Obviously the need for "balun transformers" to connect $50 \Omega$ coaxial cables at input and output is a disadvantage because these baluns set the frequency limits - but $50 \Omega / 200 \Omega$ dual-core Guanella baluns (e.g. see Sect. 1.3 in Sevick, 1990) can be made with wide bandwidth, good impedance match and low loss, and as a benefit they double the line voltage, converting the expensive transmitter power into more field strength.

Numerical simulations (Technische Universität Harburg) with the actual line geometry have shown that (assuming perfect symmetry) the fields near the line centre are sufficiently homogeneous up to $50 \mathrm{MHz}$, and the fraction of $\mathrm{rf}$ power radiated into the far field is low - approx. $0.1 \%$ of the input power at $30 \mathrm{MHz}$. The calibrations are performed by licensed radio amateurs and only at frequencies assigned to the (experimental) amateur radio service, therefore parasitic radiation is tolerable, and external room shielding of the transmission line is not mandatory. It has been verified that the homogeneous area inside the line is sufficiently large to simultaneously accomodate the small sensor of the transfer field strength meter and the radiation monitor. Both are mounted at the line axis, each with $0.15 \mathrm{~m}$ longitudinal distance from the center point. For this arrangement the interaction (scattered fields) between the sensors was found to be negligible.

During the calibrations the required traceable electric field strength is adjusted using the readout value of the transfer field strength meter. This reference instrument reads its calibration data into the control program and automatically corrects the frequency-, linearity- and temperature deviations of its sensor.

\section{Electronics and control}

Figure 2 shows the block diagram of the complete calibration system. Rf signal processing is conventional - a synthesized rf generator feeds the broadband driver and power amplifier stages, and harmonics are suppressed by a switched lowpass filter. The rf power is passing through the balanced parallel plate line via the 1:4 and 4:1 Guanella baluns, then going into a $20 \mathrm{~dB}, 50 \mathrm{ohms}$ power attenuator as the main line termination. At the attenuator output a small fraction $(1 \%)$ of the rf power is monitored with a rf voltmeter for stability, and for expanding the dynamic range of the transfer field strength meter, if required.

Most of the system components are remote-controlled, with an IEEE488-/USB-converter to connect the old-style instruments with a laptop computer. To avoid field distortions, the field strength meter(s) are isolated with plastic fiberoptics and also connected via USB.

This equipment can produce up to $120 \mathrm{~W}$ rf power, resulting in an electric field strength up to $200 \mathrm{~V} \mathrm{~m}^{-1}$ as required.

\section{Electric field calibration}

As described above, a radiation monitor calibration is actually a measurement, and the result is the calibration factor, here defined as the ratio between the traceable, predefined electric field strength $E_{\text {ref }}$ indicated by the transfer field strength meter and the monitor readout $E_{\text {cal readout }}$ :

$k_{\text {cal E }}=\frac{E_{\text {ref }}}{E_{\text {cal readout }}}$

If we assume that the radiation monitor has a digital display with sufficient resolution, shows a stable value, and the operator makes no mistake when observing the numbers, then the 


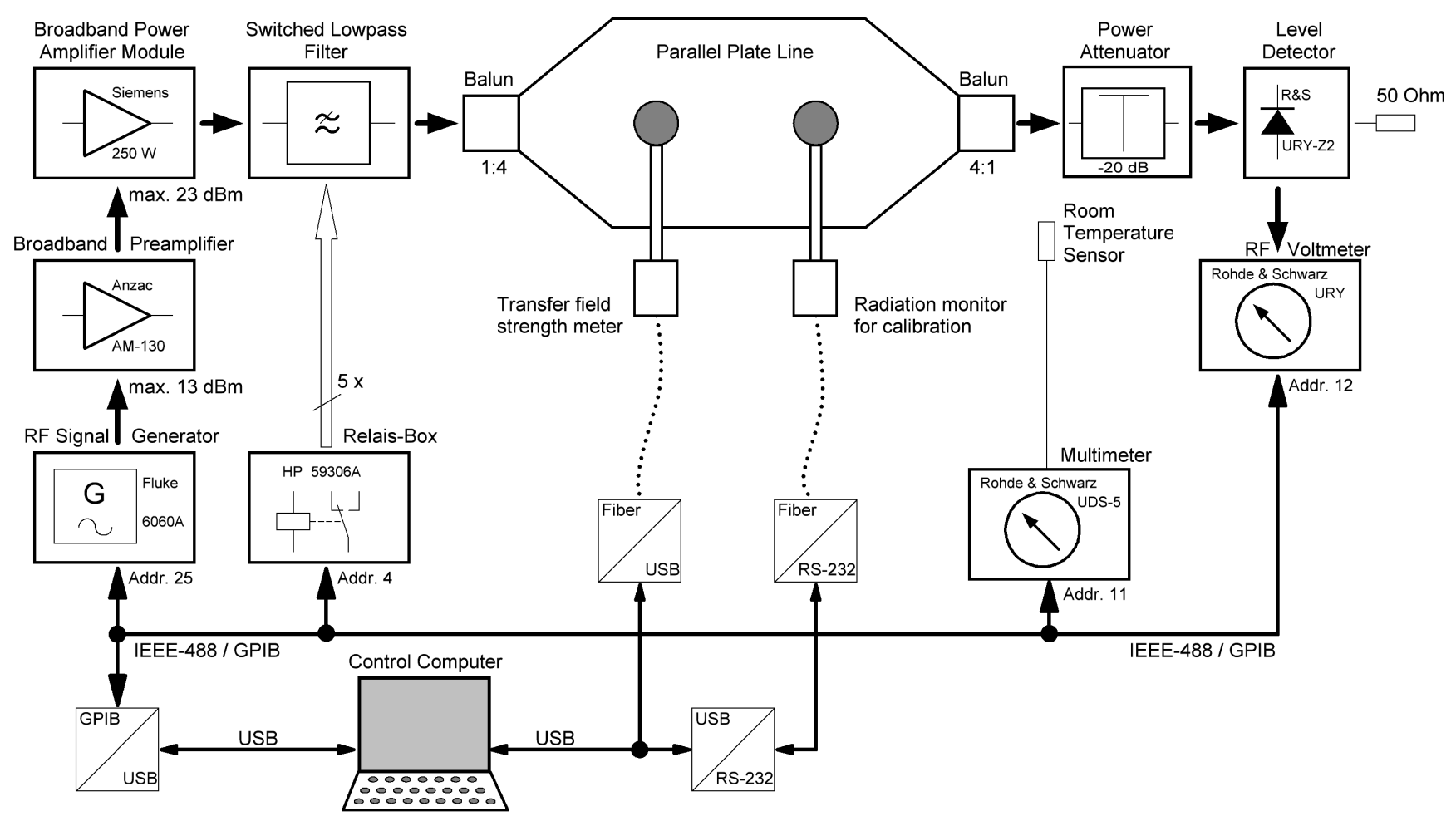

Fig. 2. Block Diagram of Calibration System.

readout value has no uncertainty. In this ideal case the uncertainty of the calibration factor from the "substitution method" would only be given by the reference value, i.e. by the uncertainty of the transfer instrument alone. Obviously the real world is different - the monitor readout probably depends on frequency, polarization, temperature etc., and may be nonlinear. The different locations of reference sensor and monitor inside the TEM line also introduce an "inhomogeneity" or position error. It would be much too elaborate investigating all these influences during an usual calibration procedure, where only the frequency $f$ and the field strength level $E$ are varied. It is therefore assumed that all other parameters are independent and can (formally) be compensated by a product of correction factors. Of course, in practice the influences of these parameters on the individual radiation monitor are unknown and their numerical correction is impossible. Therefore the best estimate for their associated correction factors is 1 , and informations about their uncertainty contributions must come from other sources. With all these arguments, the "model equation" for the calibration factor is:

$k_{\text {cal }}(f, E)=k_{\text {Polariz }} \cdot k_{\text {Temp }} \cdot k_{\text {inhom }} \cdot \frac{E_{\text {ref }}}{E_{\text {cal readout }}}$

\section{Magnetic field calibration}

At the time of the cooperation the PTB offered no traceable rf magnetic field for calibrations, therefore a method for TEM transmission lines is described here, deriving that quantity from the traceable electric field strength $E_{\mathrm{cal}}$ established with the transfer field strength meter.

In a perfectly terminated, lossless TEM line the energy flow is constant and unidirectional, the far-field conditions are valid, and the following equation is exact, giving the corresponding magnetic field $H_{\text {cal }}$, which is therefore also traceable:

$H_{\text {cal }}=\frac{E_{\text {cal }}}{Z_{0}}$

where (in SI units) the free space impedance is $Z_{0} \approx 377 \Omega$.

In this case the traceable magnetic field strength is defined with no additional uncertainty. Although the TEM transmission line and termination used here are carefully optimized, some spurious reflections can not be completely avoided, mainly caused by the baluns, the tapered sections and the power attenuator input. Superposition of the forward and reflected waves produces a "standing wave" pattern along the non-ideal TEM-line, and therefore the ratio of the field amplitudes becomes a function of the location $x$ :

$Z_{\mathrm{TEM}}(x)=\frac{E(x)}{H(x)}$ 
Formally this is taken into account by introducing a correction factor $k_{X}$ for the (local) line impedance into (Eq. 3), valid at a certain location $x$ :

$H_{\mathrm{cal}}(x)=\frac{E_{\mathrm{cal}}(x)}{Z_{\mathrm{TEM}}(x)}=\frac{E_{\mathrm{cal}}(x)}{Z_{0} \cdot k_{x}}$

Without detailed informations about the reflections the function $k_{X}$ along the line axis remains unknown, and a numerical correction is impossible. So we have to set $k_{X}=1$ (or $Z_{\mathrm{TEM}}=Z_{0}$ ) for all values of $x$ as the best estimate and must accept an additional uncertainty $u_{\text {TEM }}$ instead. Therefore the magnetic calibration field has a larger uncertainty than the electric field, and to maintain traceability, this uncertainty contribution must be determined from the reflection coefficient of the imperfect line.

For simplicity the reflection coefficient $r$ is defined here as the ratio between the forward and reverse wave amplitudes, a real number neglecting the phase relations between these waves.

Usual network analyzers can be configured to measure small one-port reflection coefficients, if calibrated with a precision line termination. Their readout $a$ is often specified as "return loss" in decibels, with $a=20 \mathrm{~dB} \cdot \lg (r)$, and correspondingly for given $a$ the reflection coefficient is $r=10 \frac{a}{20 \mathrm{~dB}}$ The line discussed here has a measured input return loss below $-30 \mathrm{~dB}$ over the complete frequency range from $1.8 \mathrm{MHz}$ to $30 \mathrm{MHz}$, when terminated with its power attenuator. The corresponding reflection coefficient is therefore below $r=0.032$ and we shall use this value later as the worst case over that complete frequency range.

With the measured reflection coefficient the extreme values of the $E$ and $H$ field strength are calculated as:

$E_{\max }=E_{\mathrm{cal}}(1+r)$

and

$E_{\min }=E_{\mathrm{cal}}(1-r)$

$H_{\text {max }}=H_{\text {cal }}(1+r)$

and

$H_{\min }=H_{\text {cal }}(1-r)$

Along a lossless line the energy density is constant, therefore $H_{\max }$ is at the same location as $E_{\min }$ and vice versa. The reflection coefficient then gives the limits for $Z_{\mathrm{TEM}}(x)$ :

$Z_{\mathrm{TEM}, \min }=\frac{E_{\min }}{H_{\max }}=\frac{E_{\mathrm{cal}}(1-r)}{H_{\mathrm{cal}}(1+r)}$

$Z_{\mathrm{TEM}, \max }=\frac{E_{\mathrm{max}}}{H_{\text {min }}}=\frac{E_{\mathrm{cal}}(1+r)}{H_{\mathrm{cal}}(1-r)}$

With total reflection $(r=1) Z_{\mathrm{TEM}}$ varies between 0 and infinity, but for $r \ll 1$ the approximations

$1+r \approx \frac{1}{1-r}$ and

$1-r \approx \frac{1}{1+r}$

are useful to "linearize" the expressions for the extremes by neglecting square terms of $r$ :

$Z_{\mathrm{TEM}, \min } \approx Z_{0}(1-r)^{2}=Z_{0}\left(1-2 r+r^{2}\right) \approx Z_{0}(1-2 r)$

$Z_{\mathrm{TEM}, \max } \approx Z_{0}(1+r)^{2}=Z_{0}\left(1+2 r+r^{2}\right) \approx Z_{0}(1+2 r)$

These extremes restrict the values of the unknown function $k_{X}$ in Eq. (5) to the interval $\{1 \pm 2 r\}$, therefore the best possible match within the transmission line system is essential for lowest uncertainty of the magnetic calibration field.

The complete "model equation" for the $H$-field monitor calibration is (with the same arguments as for the $E$ field calibration factor):

$k_{\text {cal }}(f, H)=k_{\text {Polariz }} \cdot k_{\text {Temp }} \cdot k_{\text {inhom }} \cdot \frac{E_{\text {cal }}}{Z_{0} \cdot k_{X} \cdot H_{\text {readout }}}$

Along a standing-wave pattern large deviations from the mean values of voltage or field strength are more probable than small ones, therefore an U-shaped distribution for the additional parameter $k_{X}$ must be assumed to calculate the variance.

\section{Calibration procedure and certificate}

A computer program operates the rf signal generator, lowpass filter, rf voltmeter, reference field strength meter, and the device to be calibrated, if it has a suitable interface. Additionally, it assembles and stores all relevant data for later processing. After proper setup of the complete system hardware, the program makes nearly simultaneous measurements with the traceable transfer instrument, the rf voltmeter and (if possible) the radiation monitor to be calibrated, scanning all devices at the fastest possible rate. The operator can manually preset the frequency and field strength and immediately see the results. This manual mode enables a detailed function check and individual calibration measurements. For the radiation meter types used by the DARC, an automatic calibration sequence is implemented in the program, setting three different $E$ - or $H$-field strength levels (depending on the field probe type) for each of the shortwave amateur radio bands. For each setting the frequency, the field strength at the transfer instrument, the readout from the device under calibration and the rf voltage across the line are written into a data file as documentation of the original raw data.

Final processing is done by transferring these raw data into a prepared Excel spreadsheet template for calculating the calibration coefficient $(s)$, preparing the tables and diagrams, and finally printing these results as the attachment for the DARC calibration certificate. 


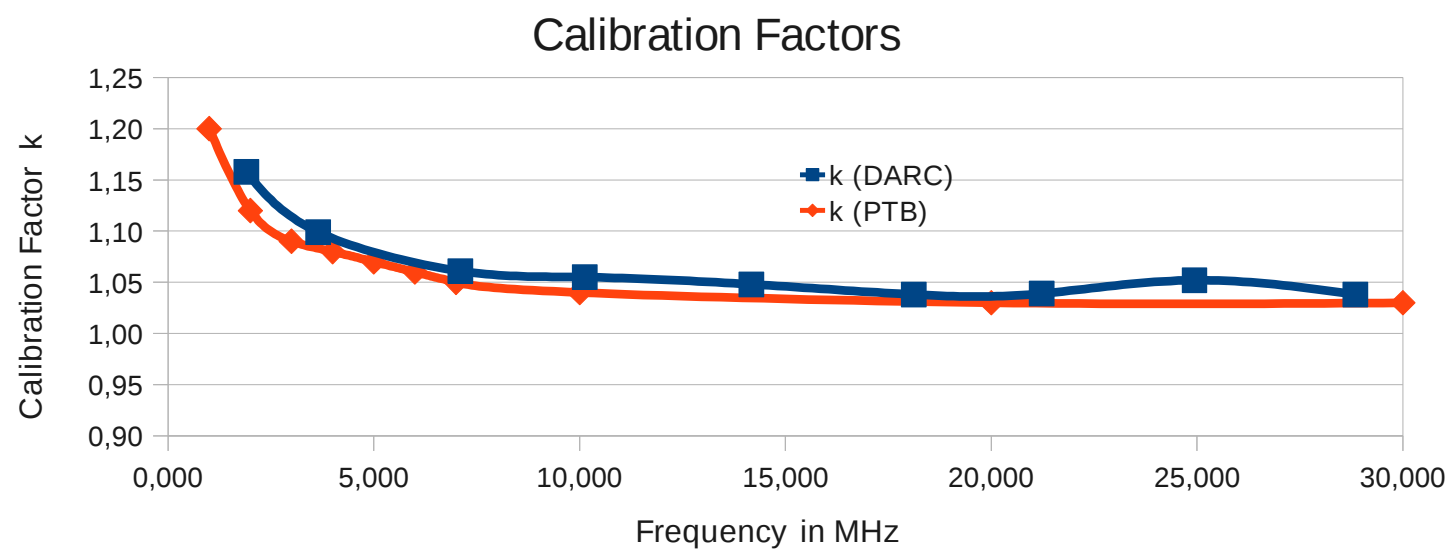

Fig. 3. Comparison of Calibration Factors.

\section{Measurement results, comparison with PTB results}

For one of the DARC radiation monitors two calibration certificates were available for direct comparison, one from the $\mathrm{PTB}$, and the second from the DARC, obtained with the procedure described here. The PTB gives only $E$ field calibration data from a large "GTEM" cell, but for three orthogonal orientations of the monitor, while the DARC specifies data for $E$ and $H$ fields, but for only one monitor position. Therefore the data for the $E$ field sensor with the monitor axis parallel to the magnetic field vector were chosen. It should be noted that the traceability of both calibrations is based on the $\mu$ TEM cell at the PTB, but the calibrations were performed in different transmission line types and with independent transfer field strength meters. Although the calibration factors in Fig. 3 are therefore partially correlated, they confirmthe consistency of the calibration fields within a few percent, and they demonstrate the stability of all devices over one year. Additionally, the actual calibration factors are still close to the initial adjustment of the radiation monitor at the factory many years earlier.

\section{Conclusions}

The results demonstrate that it is possible to calibrate radiation monitors for the electric and magnetic field strength, traceable to the SI units, with a reasonably low uncertainty, without compromising quality, and with low-cost equipment available to radio amateurs. 


\section{Appendix A}

\section{Uncertainty budgets for $E$ and $\boldsymbol{H}$ field calibrations}

The calculation of the calibration factor uncertainties follows the widely accepted concept in the "Guide to the expression of uncertainty in measurement" (GUM) (BIPM, 2008). Based on a "model equation" describing the measurement or calibration process, the variances of all relevant input parameters are summed, and an overall uncertainty for the result parameter is calculated. Here we avoid a detailed derivation of the formulas and use an educational version of the "GUM Workbench" program (Metrodata GmbH, 2012) that implements the rules of the GUM. The Eqs. (2) and (12) are taken as the model equations for the corresponding uncertainty budgets, shown here as the program output.

\section{A1 DARC-Procedure: E-Field Sensor Calibration}

This is the uncertainty budget for the calibration of radiation monitors used to ensure public safety in the proximity of amateur radio stations. These instruments are calibrated with an electric field inside an open parallel-plate TEM transmission line following the "substitution method", described by the model Eq. (2) explained in the publication text. The result is the calibration factor as the ratio between the field strength generated inside the transmission line and the readout from the instrument to be calibrated.
Model equation:

$k_{\mathrm{cal}}=k_{\mathrm{pol}} \cdot k_{\mathrm{Temp}} \cdot k_{\text {inhom }} \cdot E_{\mathrm{cal}} / E_{\text {readout }}$

Input data are listed in Table A1, for calculated uncertainty budget see Table A3.

\section{A2 DARC-Procedure: H-Field Sensor Calibration}

This is the uncertainty budget for the calibration of radiation monitors used to ensure public safety in the proximity of amateur radio stations. These instruments are calibrated with a magnetic field inside an open parallel-plate TEM transmission line following the "substitution method", described by the model Eq. (12) explained in the publication text. The result is the calibration factor as the ratio between the field strength generated inside the transmission line and the readout from the instrument to be calibrated.

Model equation:

$k_{\text {cal }}=k_{\text {pol }} \cdot k_{\text {Temp }} \cdot k_{\text {inhom }} \cdot E_{\text {cal }} /\left(Z_{0} \cdot k_{X} \cdot H_{\text {readout }}\right)$

Input data are listed in Table A2, for calculated uncertainty budget see Table A4. 
Table A1. List of Quantities.

\begin{tabular}{lll}
\hline Quantity & Unit & Definition \\
\hline$k_{\text {cal }}$ & & $\begin{array}{l}\text { calibration factor as result } \\
\text { correction factor for polarization } \\
k_{\text {pol }}\end{array}$ \\
$k_{\text {Temp }}$ & & $\begin{array}{l}\text { correction factor for temperature drift } \\
\text { correction factor for calibration field in- } \\
\text { homogeneity } \\
k_{\text {inhom }}\end{array}$ \\
$E_{\text {cal }}$ & $\mathrm{V} \mathrm{m}^{-1}$ & $\begin{array}{l}\text { electric field strength } \\
\text { readout value from radiation monitor }\end{array}$ \\
$E_{\text {readout }}$ & $\mathrm{V} \mathrm{m}^{-1}$ & \\
\hline
\end{tabular}

$k_{\text {pol }}$ : Type B normal distribution

Value: 1 Expanded Uncertainty: 0.05

Coverage Factor: 2 correction factor for different or unknown field polarization, here no numerical correction is made because it is assumed that the instrument is used in proper orientation. Its uncertainty is estimated.

$k_{\text {Temp: }}$ Type B rectangular distribution

Value: 1 Halfwidth of Limits: 0.05 correction factor for temperature drift, if the monitor is used within the temperature range specified by the manufacturer. No numerical correction possible, uncertainty specified by manufacturer without further details, therefore rectangular distribution assumed.

$k_{\text {inhom: }}$ : Type B normal distribution

Value: 1 Expanded Uncertainty: 0.02

Coverage Factor: 2 correction factor for possible field inhomogeneity between transfer sensor and radiation monitor positions. No numerical correction possible, uncertainty estimated from numerical field calculations.

$E_{\text {cal }}$ : Type B normal distribution

Value: $20 \mathrm{~V} \mathrm{~m}^{-1}$

Expanded Uncertainty: $2 \mathrm{~V} \mathrm{~m}^{-1}$

Coverage Factor: 2 traceable electric field strength in $\mathrm{V} \mathrm{m}^{-1}$ generated inside TEM

transmission line, as indicated by the PTB transfer field strength meter. Uncertainty

taken from the PTB calibration certificate.

$E_{\text {readout }}$ : Type B normal distribution

Value: $20 \mathrm{~V} \mathrm{~m}^{-1}$

Expanded Uncertainty: $0.01 \mathrm{~V} \mathrm{~m}^{-1}$

Coverage Factor: 1 readout value in $\mathrm{V} \mathrm{m}^{-1}$ from digital display of radiation monitor to be calibrated, assumed to be stable and accurate.
Table A2. List of Quantities.

\begin{tabular}{|c|c|c|}
\hline Quantity & Unit & Definition \\
\hline$k_{\mathrm{cal}}$ & & calibration factor as result \\
\hline$k_{\mathrm{pol}}$ & & correction factor for polarization \\
\hline$k_{\text {Temp }}$ & & correction factor for temperature drift \\
\hline$k_{\text {inhom }}$ & & $\begin{array}{l}\text { correction factor for calibration field in- } \\
\text { homogeneity }\end{array}$ \\
\hline$E_{\text {cal }}$ & $\mathrm{V} \mathrm{m}^{-1}$ & electric field strength \\
\hline$Z_{0}$ & Ohm & free space impedance (exact) \\
\hline$k_{X}$ & & $\begin{array}{l}\text { correction factor for local } \\
\text { line impedance }\end{array}$ \\
\hline$H_{\text {readout }}$ & $\mathrm{A} / \mathrm{m}$ & magnetic field strength \\
\hline
\end{tabular}

$k_{\text {pol }}$ : Type B normal distribution

Value: 1

Expanded Uncertainty: 0.05

Coverage Factor: 2 correction factor for different or unknown field polarization, here no numerical correction is made because it is assumed that the instrument is used in proper orientation. Its uncertainty is estimated.

$k_{\text {Temp }}$ : Type B rectangular distribution

Value: 1 Halfwidth of Limits: 0.05

correction factor for temperature drift, if the monitor is used within the temperature range specified by the manufacturer. No numerical correction possible, uncertainty specified by manufacturer without further details, therefore rectangular distribution assumed.

$k_{\text {inhom: }}$ Type B normal distribution

Value: 1 Expanded Uncertainty: 0.02

Coverage Factor: 2 correction factor for possible field inhomogeneity between transfer sensor and radiation monitor positions. No numerical correction possible, uncertainty estimated from numerical field calculations.

$E_{\text {cal }}$ : Type B normal distribution

Value: $20 \mathrm{~V} \mathrm{~m}^{-1}$

Expanded Uncertainty: $2 \mathrm{~V} \mathrm{~m}^{-1}$

Coverage Factor: 2 traceable electric field strength in $\mathrm{V} \mathrm{m}^{-1}$ generated inside TEM transmission line, as indicated by the PTB transfer field strength meter. Uncertainty taken from the PTB calibration certificate.

$Z_{0}$ : Constant

Value: $377 \mathrm{Ohm}$

$k_{X}$ : Type B U-shaped distribution

Value: 1

Halfwidth of Limits: 0.064

correction factor for local line impedance. No numerical correction possible, limits of $\mathrm{U}$-shaped distribution from reflection coefficient measurement

$\mathrm{H}_{\text {readout }}$ : Type B normal distribution

Value: $0.053503 \mathrm{~A} / \mathrm{m}$ Expanded Uncertainty: $5.3503 \times 10^{-4} \mathrm{~A} / \mathrm{m}$

Coverage Factor: 1 readout value from radiation monitor 
Table A3. Uncertainty Budget for E-field Calibration $k_{\text {cal }}$ : calibration factor as result

\begin{tabular}{|c|c|c|c|c|c|c|}
\hline Quantity & Value & $\begin{array}{r}\text { Standard } \\
\text { Uncertainty }\end{array}$ & Distribution & $\begin{array}{l}\text { Sensitivity } \\
\text { Coefficient }\end{array}$ & $\begin{array}{l}\text { Uncertainty } \\
\text { Contribution }\end{array}$ & Index \\
\hline$k_{\text {pol }}$ & 1.0000 & 0.0250 & normal & 1.0 & 0.025 & $15.4 \%$ \\
\hline$k_{\mathrm{Temp}}$ & 1.0000 & 0.0289 & rectangular & 1.0 & 0.029 & $20.5 \%$ \\
\hline$k_{\text {inhom }}$ & 1.0000 & 0.0100 & normal & 1.0 & 0.010 & $2.5 \%$ \\
\hline $\mathrm{E}_{\mathrm{cal}}$ & $20.00 \mathrm{~V} \mathrm{~m}^{-1}$ & $1.00 \mathrm{~V} \mathrm{~m}^{-1}$ & normal & 0.050 & 0.050 & $61.6 \%$ \\
\hline $\begin{array}{l}E_{\text {readout }} \\
k_{\text {cal }}\end{array}$ & $\begin{array}{r}20.0000 \mathrm{~V} \mathrm{~m}^{-1} \\
1.0000\end{array}$ & $\begin{array}{r}0.0100 \mathrm{~V} \mathrm{~m}^{-1} \\
0.0637\end{array}$ & normal & -0.050 & $-500 \times 10^{-6}$ & $0.0 \%$ \\
\hline \multicolumn{7}{|c|}{ Calibration factor as result } \\
\hline $\begin{array}{l}\text { Results: } \\
\text { Quantity }\end{array}$ & Value & $\begin{array}{r}\text { Expanded } \\
\text { Uncertainty }\end{array}$ & $\begin{array}{r}\text { Coverage } \\
\text { factor }\end{array}$ & Coverage & & \\
\hline$k_{\mathrm{cal}}$ & 1.00 & 0.13 & 2.00 & $95 \%$ (normal) & & \\
\hline
\end{tabular}

Table A4. Uncertainty Budget for H-field Calibration $k_{\text {cal }}$ : calibration factor as result.

\begin{tabular}{|c|c|c|c|c|c|c|}
\hline Quantity & Value & $\begin{array}{r}\text { Standard } \\
\text { Uncertainty }\end{array}$ & Distribution & $\begin{array}{l}\text { Sensitivity } \\
\text { Coefficient }\end{array}$ & $\begin{array}{l}\text { Uncertainty } \\
\text { Contribution }\end{array}$ & Index \\
\hline$k_{\text {pol }}$ & 1.0000 & 0.0250 & normal & 0.99 & $0.0250(\mathrm{rel})$ & $10.1 \%$ \\
\hline$k_{\text {Temp }}$ & 1.0000 & 0.0289 & rectangular & 0.99 & 0.0289 (rel) & $13.4 \%$ \\
\hline$k_{\text {inhom }}$ & 1.0000 & 0.0100 & normal & 0.99 & 0.0100 (rel) & $1.6 \%$ \\
\hline$E_{\text {cal }}$ & $20.00 \mathrm{~V} \mathrm{~m}^{-1}$ & $1.00 \mathrm{~V} \mathrm{~m}^{-1}$ & normal & 0.050 & 0.0500 (rel) & $40.2 \%$ \\
\hline $\mathrm{Z}_{0}$ & $377.0 \mathrm{Ohm}$ & & & & & \\
\hline$k_{X}$ & 1.0000 & 0.0453 & U-distr. & -0.99 & -0.0453 (rel) & $33.1 \%$ \\
\hline$H_{\text {readout }}$ & $0.053503 \mathrm{~A} / \mathrm{m}$ & $535 \times 10^{-6} \mathrm{~A} / \mathrm{m}$ & normal & -19 & -0.0100 (rel) & $1.6 \%$ \\
\hline$k_{\mathrm{cal}}$ & 0.9915 & 0.0782 & & & & \\
\hline \multicolumn{7}{|c|}{ Calibration factor as result } \\
\hline \multicolumn{7}{|l|}{ Results: } \\
\hline Quantity & Value & $\begin{array}{l}\text { Expanded } \\
\text { Uncertainty }\end{array}$ & $\begin{array}{r}\text { Coverage } \\
\text { factor }\end{array}$ & Coverage & & \\
\hline$k_{\mathrm{cal}}$ & 0.99 & 0.16 & 2.00 & $95 \%$ (normal) & & \\
\hline
\end{tabular}


Acknowledgements. The authors wish to thank U. Wintzer (DL7FZ) for making and optimizing the transmission line and the baluns, for assembling the complete system hardware and a lot of measurements. More thanks go to all other members of the DARC "ad-hoc calibration group" - W. Schlink (DL3OAP), K. Stamm (DL6CY), D. Vieth (DJ7GD) - for their continuing interest, support, critical discussions and a lot of ideas.

\section{References}

Bundesnetzagentur für Elektrizität, Gas, Telekommunikation, Post und Eisenbahnen (BNetzA): BEMFV - Verordnung zur Beschränkung elektromagnetischer Felder (www.bundesnetzagentur.de), 2002.

BNetzA: Anleitung zur Durchführung der Anzeige ortsfester Amateurfunkanlagen nach $\S 9$ der Verordnung zur Beschränkung elektromagnetischer Felder (BEMFV), 2002.

Crawford M. L.: Generation of standard EM fields using TEM transmission cells, IEEE Trans. EMC, EMC-16, 189-195, 1974.

Glimm J., Münter K., Pape R., Schrader T., and Spitzer M.: The New National Standard of EM Field Strength, Realisation and Dissemination, Proc 12th Int Symp on EMC, Zürich, Switzerland, 611-613, ISBN 3-9521199-1-1, 1997.
Münter, K., Pape, R., and Glimm, J.: Portable E-Field strength meter system and its traceable calibration up to $1 \mathrm{GHz}$ using a $\mu$ TEM cell; 1997, IEEE Trans. Instr. Meas, IM-46, 549-550, 1997.

Sevick, J. (W2FMI): Transmission Line Transformers; 2nd edn., ARRL - American Radio Relay League, 1990.

Technische Universität Harburg, Institut für Theoretische Elektrotechnik: Program "Concept II", http://www.tet.tu-harburg.de/ concept/index.de.html, last access: 15.02.2012, 2012.

Calibration Certificate DARC Nr. 104, dated 2009.11.25 and Calibration Certificate PTB Sign 21059 PTB 10, dated: 2010.11.17, 2010.

BIPM - Bureau International des Poids et Mesures: Evaluation of measurement data - Guide to the expression of uncertainty in measurement (GUM); 1st edn., Paris, Sept. 2008, download from http://www.bipm.org/en/publications/guides/gum.html, last access: 18.01.2012, 2012.

Metrodata GmbH: available at: www.metrodata.de, last access: 15.02.2012, 2012. 